\title{
Survival prediction with score model based on clinical characteristics in advanced HCC patients receiving oxaliplatin-containing regimens
}

\author{
L. QIAN*, Y. B. DONG ${ }^{*}$, L. L. ZHAO, H. J. ZHAO, J. W. CUI, N. Y. WANG* \\ Cancer Center, the First Hospital of Jilin University, Changchun, China \\ ${ }^{*}$ Correspondence: nanyawang@yahoo.com

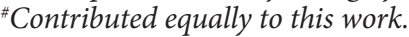

Received July 6, 2018 / Accepted October 17, 2018

\begin{abstract}
A score model based on clinical characteristics in advanced hepatocellular carcinoma (HCC) patients treated with systemic chemotherapy of oxaliplatin-containing regimens was established to evaluate progression-free survival (PFS) and overall survival (OS). Thirty HCC patients eligible for radical resection were involved in the retrospective study, and these were divided into the good response group (complete response (CR)/partial response (PR) and the poor response group (stable disease (SD)/progression disease (PD). The median PFS and OS were compared in the two groups. PFS and OS combined with clinical characteristics were evaluated by univariate and multivariate analyses. The score model was defined with 1 score for each characteristic, and score model cut-off values were determined by the receiver operating characteristic curve (ROC) which describes treatment response. The median PFS was 10 and 2 months $(\mathrm{p}<0.001)$, and the median OS was 13 and 4 months $(\mathrm{p}=0.011)$ for the $\mathrm{CR} / \mathrm{PR}$ and $\mathrm{SD} / \mathrm{PD}$ groups, respectively. The score of 1 was the optimal cutoff value, with sensitivity ranging from 52.6 to $63.2 \%$ and specificity from 81.8 to $100 \%$ (AUC $=0.773, \mathrm{p}=0.014$ ). The median PFS for good and poor response groups was 9 months and 1month $(\mathrm{p}<0.001)$ and the median OS was 22 and 3 months at $p<0.001$, respectively. Patients with scores above 1 had poor response, with median 3 months OS and 1 month PFS, and patients with scores of 0 and 1 established good response, with median 22 months OS and 9 months PFS, respectively.
\end{abstract}

Key words: oxaliplatin-containing regimen, HCC patients, progression-free survival (PFS), overall survival (OS), score model

Patients with advanced hepatocellular carcinoma (HCC) have poor survival rate of 6-20 months [1]. It was reported that one year survival rate was about 25\% in 1993 and $30 \%$ in 2003 [2] with improved diagnosis and multiple treatment means. The Sorafenib oral tyrosine kinase inhibitor (TKI) is standard first-line systemic therapy for HCC patients, and this improves overall survival (OS) by almost three months $[3,4]$. Although the agent is effective, it does not generally induce tumor shrinkage. The frequency of adverse events for all-grades was reported between $21 \%$ and $73 \%$, including diarrhea, weight loss, hand-foot skin reaction and hypophosphatemia $[3,5,6]$. Therefore, Sorafenib application in clinical practice is limited and a second-line agent for HCC patients is imperative.

HCC is highly refractory to systemic chemotherapy because of its heterogeneity [7] and this prompted the following combined systemic chemotherapy agents: randomized controlled FOLFOX4 with infusional fluorouracil, leucovorin, and oxaliplatin, doxorubicin in EACH [8] and retrospective gemcitabine and oxaliplatin GEMOX (also called AGEO) [9]. Results show that the oxaliplatin and gemcitabine regimens are relatively effective.

The FOLFOX4 study increased OS compared to doxorubicin (6.47 vs 4.90 months; $\mathrm{p}=0.04$ ) with higher median progression-free survival (PFS) and response rate. Although this indicates that systemic oxaliplatin-containing chemotherapy is recommended, the low objective response rate and limited survival after systemic chemotherapy remain major obstacles. It has also been suggested that clinical outcomes can be improved by using biomarkers or clinical characteristics to evaluate HCC patients and by precise choice of patient population. Moreover, future HCC research must ensure better understanding of the clinical and biologic factors affecting response and prognosis in stratification analysis and biomarker enhanced strategies [10].

Precise selection of patients for appropriate chemotherapy is a challenging issue because currently chosen patient survival varies from 2 to 12 months. Our study identified 
some patients with clinical characteristics which encouraged good response and longer survival after systemic chemotherapy, so we established a score model to predict prognosis in advanced HCC patients receiving oxaliplatin-containing regimen based on good and poor responses.

\section{Patients and methods}

Patient selection and study design. Thirty advanced HCC patients receiving oxaliplatin-containing regimens at the Cancer Center of the First Hospital of Jilin University in Changchun, China, were retrospectively analyzed from December 2013 to December 2015. The study was reviewed by the Ethical Committee of the First Hospital of Jilin University.

Inclusion criteria were: patients histologically or clinically diagnosed with un-resectable HCC; those in need of systemic therapy after failure with or without access to Sorafenib; measurable lesion according to Response Evaluation Criteria in Solid Tumors (RECIST version 1.1); 18 years old or over; Karnofsky performance score (KPS) no less than 70; stage B or $\mathrm{C}$ according to the Barcelona Clinic liver Cancer (BCLC) staging system; Child-Pugh stage A or B disease and adequate organ and marrow function.

Exclusion criteria were: liver transplantation, prior treatment with systemic chemo-therapeutic agents except transcatheter arterial chemo-embolization (TACE) and having herbal treatment in the previous month. Figure 1 has the patient selection flow diagram and Table 1 lists patient general data, including demographics, tumor characteristics, previous treatment, HCC etiology, Child-Pugh score, $\alpha$-fetoprotein (AFP) at baseline and after treatment, treat-

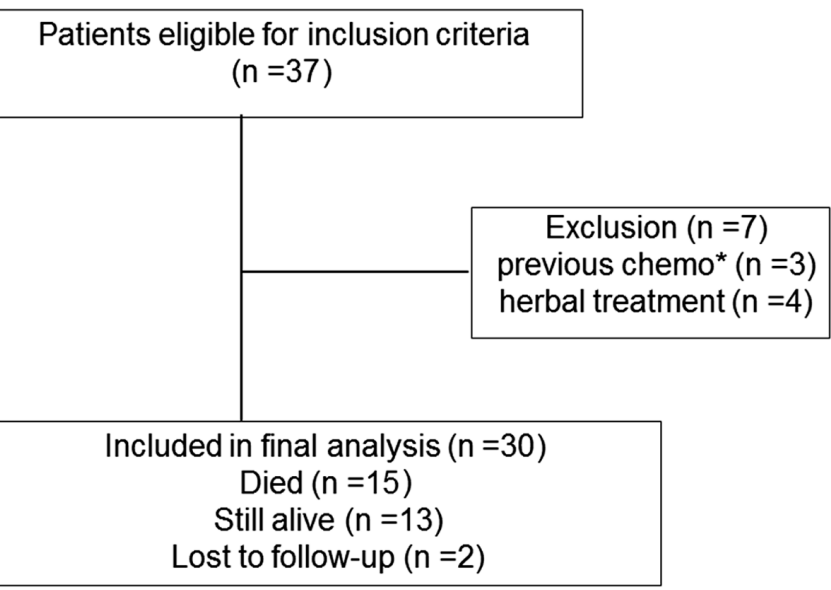

Figure 1. Flow diagram of patient selection. Eligible patients were retrospectively selected from hospital electronic medical records according to the inclusion and exclusion criteria. A total of 37 patients were inially included and 7 patients were excluded for having received previous chemotherapy $(n=3)$ and herbal treatment $(n=4)$. A total of 30 patients were included in the final analysis. At last visit, 15 patients died, 13 patients still were alive and 2 patients were lost to follow-up. chemo*: chemotherapy ment response, time to disease progression, and survival status at the last follow-up. The treatment responses included complete response (CR), partial response (PR), stable disease (SD) and progressive disease (PD) according to RESCIT criteria.

Patients were divided into $\mathrm{CR} / \mathrm{PR}$ and $\mathrm{SD} / \mathrm{PD}$ groups according to objective response after two chemotherapy courses. Survival curves evaluated the relationship between objective response and PFS and OS. The significant clinical characteristics for survival were determined by univariate and multivariate analysis and the score of 1 was defined for each significant variable. The receiver operating characteristic curve (ROC) was established for score cut-off values describing treatment response.

Table 1. The basic clinical characteristics.

\begin{tabular}{|c|c|}
\hline Basic clinical variables & Number of patients (n, \%) \\
\hline \multicolumn{2}{|l|}{ Age $(y)$} \\
\hline median & 52.5 \\
\hline range & $26-67$ \\
\hline \multicolumn{2}{|l|}{ Gender } \\
\hline male & $24(80 \%)$ \\
\hline female & $6(20 \%)$ \\
\hline \multicolumn{2}{|l|}{ Extrahepatic metastases } \\
\hline yes & $12(40 \%)$ \\
\hline no & $18(60 \%)$ \\
\hline \multicolumn{2}{|l|}{ Diffuse infusion } \\
\hline yes & $10(33 \%)$ \\
\hline no & $20(67 \%)$ \\
\hline \multicolumn{2}{|l|}{ Vascular invasion } \\
\hline yes & $13(43 \%)$ \\
\hline no & $17(57 \%)$ \\
\hline \multicolumn{2}{|l|}{ Child-Pugh scale } \\
\hline A & $23(77 \%)$ \\
\hline B & $7(23 \%)$ \\
\hline \multicolumn{2}{|l|}{ Previous treatment } \\
\hline yes & $23(77 \%)$ \\
\hline no & $7(23 \%)$ \\
\hline \multicolumn{2}{|l|}{ Combined therapy } \\
\hline yes & $6(20 \%)$ \\
\hline no & $24(80 \%)$ \\
\hline \multicolumn{2}{|l|}{ Courses of chemotherapy } \\
\hline median & 4 \\
\hline range & $4-10$ \\
\hline \multicolumn{2}{|l|}{ Decline in AFP } \\
\hline yes & $13(43 \%)$ \\
\hline no & $17(57 \%)$ \\
\hline \multicolumn{2}{|l|}{ Treatment response } \\
\hline $\mathrm{CR} / \mathrm{PR}$ & $11(37 \%)$ \\
\hline $\mathrm{SD} / \mathrm{PD}$ & $19(63 \%)$ \\
\hline \multicolumn{2}{|c|}{ Progression-free survival (m) } \\
\hline median(range) & $3.75(0-30)$ \\
\hline \multicolumn{2}{|l|}{ Overall survival (m) } \\
\hline median(range) & $8(1-26)$ \\
\hline
\end{tabular}


Treatment strategy. Patients were treated with oxaliplatin-containing regimens (FOLFOX4: oxaliplatin $85 \mathrm{mg} / \mathrm{m}^{2}$ intravenously on day 1 ; leucovorin $200 \mathrm{mg} / \mathrm{m}^{2} \mathrm{IV}$ from hour 0 to 2 on days 1 and 2 and fluorouracil $1000 \mathrm{mg} / \mathrm{m}^{2}$ on days 1 and 2 each fortnight; or gemcitabine plus oxaliplatin. Alternatively, patients were treated with gemcitabine $1000 \mathrm{mg} /$ $\mathrm{m}^{2}$ on day 1 followed by oxaliplatin $100 \mathrm{mg} / \mathrm{m}^{2}$ as a 2-hour infusion on day 2. Treatments were continued until disease progression, intolerable toxicity or patient refusal. If toxicity response with grade 4 occurred once or with grade 3-4 occurred twice, the subsequent cycle was administered only after toxicity response abated, and drug doses were then decreased approximately $20 \%$. Sorafenib was given to some patients in combination with oxaliplatin-containing regimens. All patients received organ protection agents such as proton pump inhibitors for gastric mucosa and glycyrrhizic acid for liver function.

Follow-up. All patients were monitored by serum AFP, computed tomography (CT) and/or magnetic resonance imaging (MRI), and disease status was evaluated every 4-course treatment or in 1 to 3 months after the last chemotherapy, and also earlier in patients with suspected disease progression. The PFS was defined from the time of initiating chemotherapy to disease progression or death, and OS was the period between chemotherapy and death or between chemotherapy and the last follow-up in surviving patients. All data was recorded at survivor final follow-ups, and these were completed on April 6th, 2016.

Model score assessment. We chose the common factors associated with PFS and OS, and defined the score of 1 for each factor. The correlation between scores and survival (PFS and OS) was evaluated by Kaplan-Meier curve and cut-off model score values were determined by the ROC curve which described treatment response.

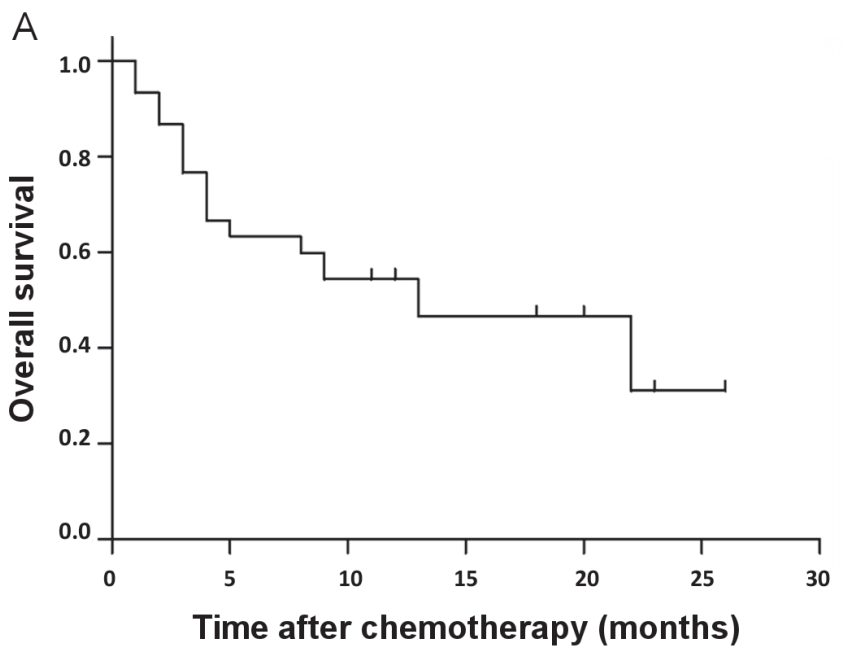

Statistical analysis. Statistical analysis was performed in SPSS 17.0 software (SPSS, Chicago, IL). Cumulative survival time was calculated by the Kaplan-Meier method and compared with the log-rank test. Univariate and multivariate Cox proportional hazard regression models estimated the hazard ratio (HR) and 95\% confidence interval (CI). Secondary analysis then assessed relationships between response and clinical characteristics. Score cutoff values predicting chemotherapy response were obtained from the ROC analysis and Fisher's exact test compared individual variables because the total number of sample was less than forty; two-tailed p-value less than 0.05 was significant.

\section{Results}

Clinical characteristics of the patients. Patient characteristics are summarized in Table 1 . The median age was 52.5 years with $26-67$ range, and 24 of the 30 patients were male. The BCLC staging classification identified that all patients were in stage C; 12 (40\%) patients had extrahepatic metastases, 10 (33\%) had diffuse infusion and 13 (33\%) had vascular invasion. Liver function indicated that $23(77 \%)$ had Chil-pugh A and the others had Chil-pugh B. Before initiating systemic chemotherapy, 23 (77\%) patients had received previous treatment, including surgery, radio-frequency ablation (RFA), TACE or Sorafenib. In addition, 6 of the 30 patients received Sorafenib in combined treatment of median 4 courses systemic chemotherapy.

During follow-up, we observed that 13 patients (43\%) had AFP decline (decreased $\geq 50 \%$ ) or kept AFP at a low level $(10 \mathrm{ng} / \mathrm{ml})$, and 11 patients (37\%) had objective response. The median OS and PFS of the patients were 13 months (1-26) and 5 months (1-21), respectively (Figure 2), and the median follow-up period was 6 months (range 1-26) and 15

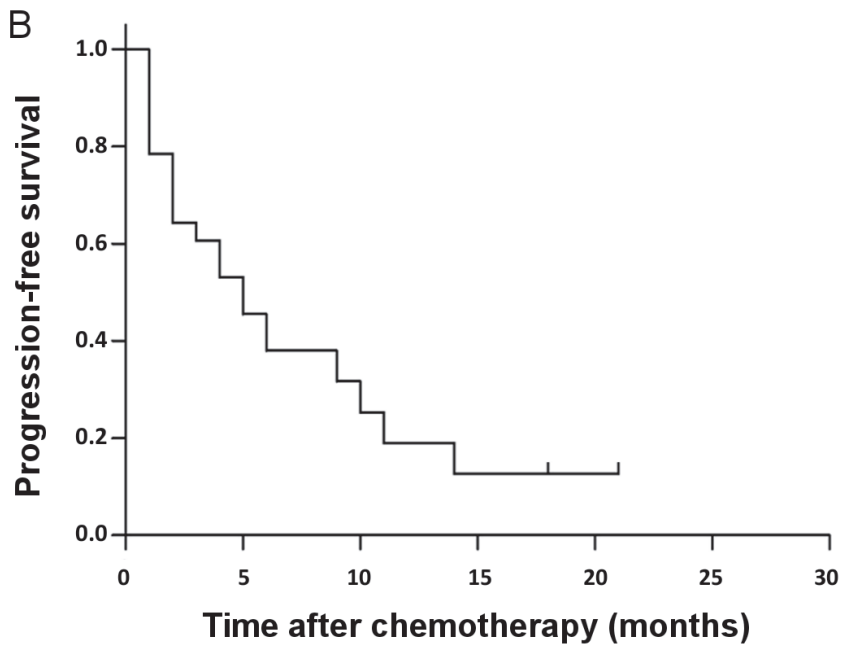

Figure 2. A) Kaplan-Meier estimates of overall survival (OS) for all patients. All included patients $(\mathrm{n}=30)$ were analyzed for overall survival using Kaplan-Meier method. The median OS was 13 months, ranging from 1 to 26 months. B) Kaplan-Meier estimates of progression-free survival (PFS) for all patients. All included patients $(n=30)$ were analyzed for progression-free survival using Kaplan-Meier method. The median PFS was 5 months, ranging from 1 to 26 months. 

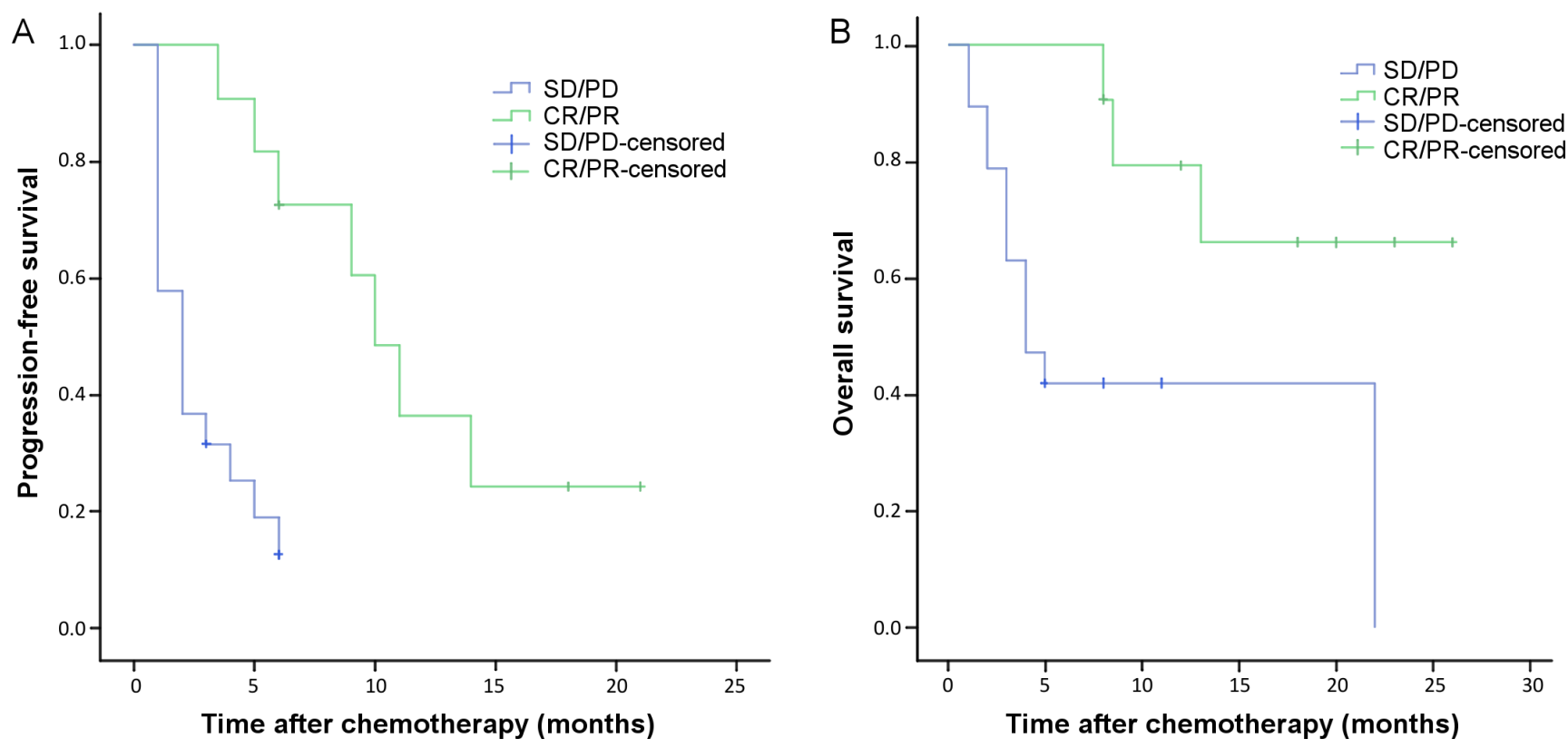

Figure 3. A) Kaplan-Meier estimates of PFS according to treatment response. The whole study population (n=30) were divided into two groups according to treatment response. For CR/PR group, it showed that the median PFS was 10 months. For SD/PD group, it showed that the median PFS was 2 months $(p<0.001)$. CR: complete response PR: partial response SD: stable disease PD: progression disease. B) Kaplan-Meier estimates of overall survival $(\mathrm{OS})$ according to the treatment response. The entire study population $(\mathrm{n}=30)$ was divided into two groups according to treatment response. For the CR/PR group, it showed that the median OS was 13 months. For the SD/PD group the median OS was 4 months $(p=0.001)$.

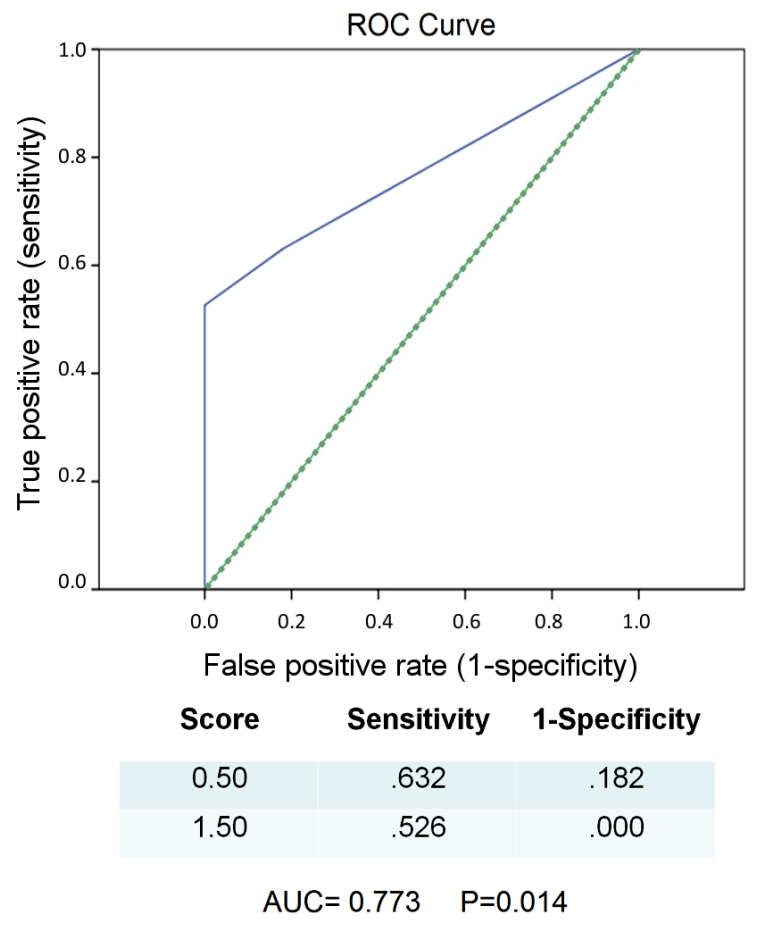

Figure 4. Receiver operating characteristic curve scores for treatment response, with cut-off value for predicting treatment responses using this ROC method. The maximum Youden index was obtained if 1.5 scores are used as cut-off value (sensitivity 0.526 ; specificity $100 \%$ ). Area under curve (AUC) was 0.773 ( $\mathrm{p}=0.014)$. patients died and 5 patients had disease progression during this period.

Treatment response and median survival. The effects of treatment responses on survival (PFS and OS) were plotted by Kaplan-Meier (Figure 3). The median PFS for the CR/PR and SD/PD groups was 10 months and 2 months $(\mathrm{p}<0.001)$, and the median OS was 13 and 4 months $(\mathrm{p}=0.011)$.

Score model and PFS/OS analysis. The clinically determining factors for PFS and OS were first evaluated with univariate analysis. The variables of previous treatment $(\mathrm{p}=0.017 ; \mathrm{p}=0.002)$, vascular invasion $(\mathrm{p}=0.001: \mathrm{p}=0.001)$ and diffuse infusion $(\mathrm{p}<0.001 ; \mathrm{p}<0.001)$ highlighted significant differences, and this was then evaluated by multivariate analyses. Changes in AFP recorded significance for PFS $(\mathrm{p}=0.034)$ but not for OS ( $\mathrm{p}=0.185$, Tables 2 and 3$)$.

Variables in diffuse infusion and vascular invasion in previous treatments were significantly different in the $\mathrm{CR} / \mathrm{PR}$ and $\mathrm{SD} / \mathrm{PD}$ groups, and this was associated with poor prognosis in univariate analyses. Prognostic score was therefore based on those three factors. To establish the model, we defined that each factor had a value of 1 , and scores were determined as the sum of the values of the factors; 16 patients had a score of 0,4 patients had 1,4 patients had 2 and 6 patients scored 3 .

ROC determined the cut-off score for evaluating treatment responses. When 1.5 was used as the cut-off value, it provided 0.526 sensitivity $100 \%$ specificity and obtained the maximum Youden index (Figure 4). The PFS and OS curves 
Table 2. Univariate and multivariate analysis for PFS.

\begin{tabular}{|c|c|c|c|c|}
\hline Clinical variables & $\begin{array}{c}\text { Univariate } \\
\text { HR }(95 \% \mathrm{CI})\end{array}$ & p-value & $\begin{array}{l}\text { Multivariate } \\
\text { HR }(95 \% \mathrm{CI})\end{array}$ & p-value \\
\hline Extrahepatic metastasis & $2.214(0.888-5.518)$ & 0.088 & & \\
\hline Previous treatment & $3.386(1.248-9.189)$ & 0.017 & $0.671(0.198-2.277)$ & 0.522 \\
\hline Combined therapy & $2.280(0.736-7.059)$ & 0.153 & & \\
\hline Child-pugh scale & $1.828(0.687-4.860)$ & 0.227 & & \\
\hline Vascular invasion & $0.181(0.066-0.497)$ & 0.001 & $1.864(0.205-16.942)$ & 0.580 \\
\hline Diffuse infusion & $0.045(0.009-0.216)$ & $<0.001$ & $1.864(0.205-16.942)$ & 0.580 \\
\hline Chang of AFP & $2.26(1.077-6.301)$ & 0.034 & $0.473(0.165-1.353)$ & 0.163 \\
\hline
\end{tabular}

Table 3. Univariate and multivariate analysis for OS

\begin{tabular}{|c|c|c|c|c|}
\hline Clinical variables & $\begin{array}{c}\text { Univariate } \\
\text { HR }(95 \% \mathrm{CI})\end{array}$ & p-value & $\begin{array}{l}\text { Multivariate } \\
\text { HR }(95 \% \mathrm{CI})\end{array}$ & p-value \\
\hline Extrahepatic metastasis & $0.394(0.141-1.102)$ & 0.76 & & \\
\hline Previous treatment & $6.575(2.050-21.088)$ & 0.002 & $2.226(0.587-8.436)$ & 0.239 \\
\hline Combined therapy & $5.037(0.657-38.616)$ & 0.120 & & \\
\hline Child-pugh scale & $0.546(0.184-1.616)$ & 0.274 & & \\
\hline Vascular invasion & $0.080(0.017-0.381)$ & 0.001 & $0.265(0.023-3.004)$ & 0.283 \\
\hline Diffuse infusion & $0.070(0.018-0.274)$ & $<0.001$ & $0.271(0.029-2.531)$ & 0.252 \\
\hline Chang of AFP & $2.079(0.705-6.135)$ & 0.185 & & \\
\hline
\end{tabular}
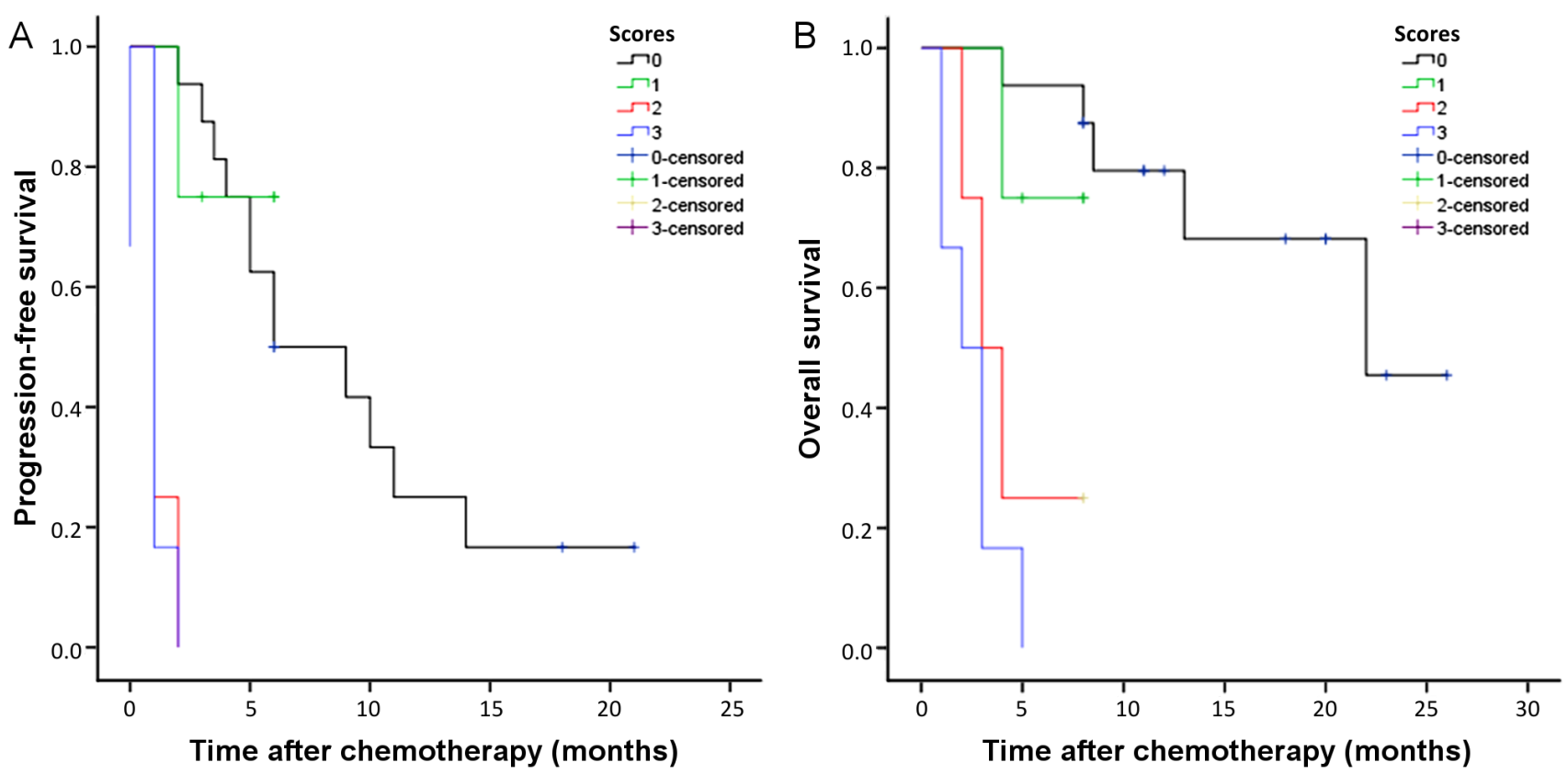

Figure 5. A) Kaplan-Meier estimates of PFS according to the scores. Each patient was given a unique score according to three clinical parameters (no previous treatment, vessel invasion and diffuse disease). The PFS curves of groups with score at $0(n=16), 1(n=4), 2(n=4)$, and 3 ( $n=6)$ were then plotted. B) Kaplan-Meier estimates of OS according to the scores. Each patient was given a unique score according to three clinical parameters (no previous treatment, vessel invasion and diffuse disease). The OS curves of groups with score at $0(n=16), 1(n=4), 2(n=4)$, and $3(n=6)$ were plotted.

were then plotted according to patient scores to establish a discriminative score model (Figure 5). Patient scores were divided; with scores above 1 in the good response group and scores between 0 and 1 indicated poor response. Thus, the 1 score was regarded as the optimal cut-off value with sensi- tivity ranging from $52.6 \%$ to $63.2 \%$ and specificity ranging from $81.8 \%$ to $100 \%$ (AUC=0.773, $\mathrm{p}=0.014$ ). The median PFS in good and poor response groups was 9 months and 1 month ( $\mathrm{p}<0.001$, Figure $6 \mathrm{~A})$, and the median OS was 22 and 3 months respectively $(p<0.001)$ (Figure $6 \mathrm{~B})$. 

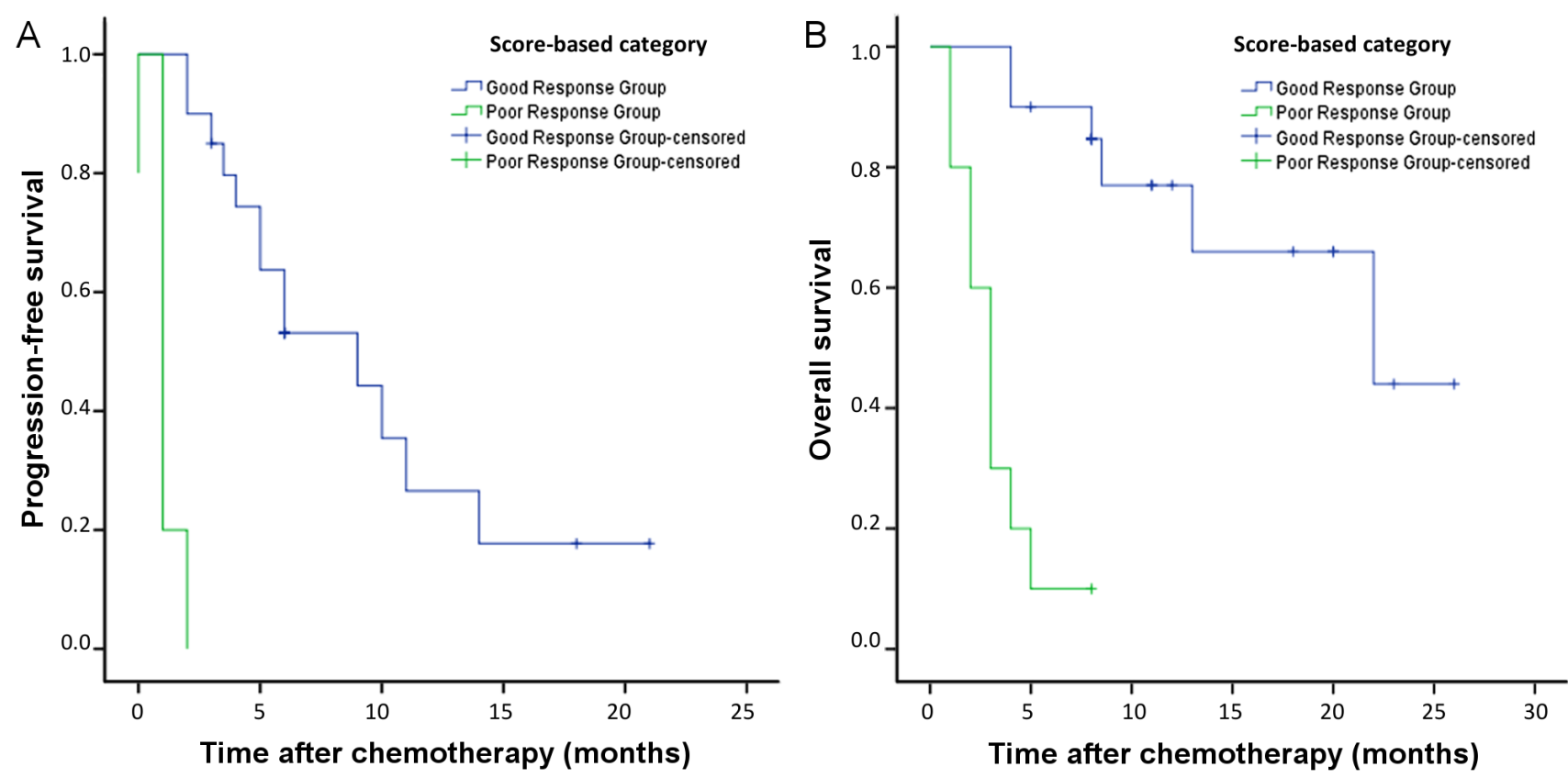

Figure 6. A) Kaplan-Meier estimates of PFS according to the 1 score-based category. The whole study population (n=30) was divided into groups with scores at $0 / 1(n=20)$ and $2 / 3(n=10)$. Kaplan-Meier showed that the median PFS were 1 month and 9 months, respectively $(p<0.001)$. B) Kaplan-Meier estimates of OS according to the 1 score-based category. The whole study population $(n=30)$ was divided into groups with scores at $0 / 1(n=20)$ and $2 / 3$ $(n=10)$. Kaplan-Meier showed that the median OS was 3 months and 22 months, respectively $(p<0.001)$.

\section{Discussion}

Our research established a score model based on three factors: previous treatment, vessel invasion, and diffuse disease in order to distinguish treatment response and survival in advanced HCC patients receiving oxaliplatincontaining regimens. The median PFS and OS were 5 and 13 months, and these results were similar to those in the AGEO study which contained the largest sample size (240 patients). This comprised GEMOX therapy strategy through a retrospective multicenter study design and showed that the median PFS and OS were 4.5 (95\% CI, 4-6) and 11 months (95\% CI, 9-14), respectively [9].

The survival data in this study is superior to that in the $\mathrm{EACH}$ study which was a prospective, international, multicenter, open-label, randomized, phase III study of FOLFOX4 versus DOX in patients with advanced HCC. It showed the median PFS and OS were 2.93 (95\% CI, 2.43-3.53) and 6.4 months (95\% CI, 5.30-7.03) in the FOLFOX4 arm, respectively [9]. The objective response rate (ORR) was $37 \%$ in our study, and $22 \%$ and $8.7 \%$ in the AGEO and $\mathrm{EACH}$ study, respectively. The noted differences may be due to the following; (i) the varied effectiveness of different regimens, though both were oxaliplatin-containing regimens; (ii) some patients had combined Sorafenib therapy in our study; (iii) different treatments for patients; for example, patients were treated with previous chemotherapy in the AGEO study and with first-line therapy in the EACH study and (iv) we contended with a small sample size and normal retrospective study drawbacks.

We also identified that some clinical characteristics associated with treatment response were reported in other studies. One study, which enrolled 147 untreated HCC patients, showed that patients with significant cirrhosis, performance status of $2-3$, tumor in over $50 \%$ of the liver and tumor thrombus in the main portal trunk may not respond to chemotherapy [11]. A further study showed that lack of radiologically active intra-hepatic disease was an independent favorable prognostic factor in metastatic HCC patients receiving systemic chemotherapy [12]. This implies that liver tumor size influences patient outcome.

It is interesting that the patients without previous treatment had worse response to chemotherapy. One possible reason is that hepatocarcinoma in patients initially diagnosed with HCC in advanced stages, without the opportunity of local or radical treatment, can be very aggressive and refractory to chemotherapy. This phenomenon requires further exploration.

Our previous results [13] revealed that the combination of Sorafenib and chemotherapy may not improve the clinical outcome. A randomized phase II trial comparing Sorafenib alone with Sorafenib plus GEMOX demonstrated that the main objective (4-month PFS rate $>50 \%$ ) was achieved but was comparable in both arms (54\% and $64 \%$ respectively), and median PFS and OS were not significantly different in the two arms. A similar trial showed that median progression 
and OS were not significantly different between Sorafenib alone and the combined GEMOX arm. That suggests that the addition of Sorafenib to cytotoxic chemotherapy can provide only modest synergism/additive effect [14].

We also noted that previous treatment variables, vascular invasion, diffuse infusion and AFP changes significantly influenced PAS and OS in univariate compared to multivariate regression analysis (Tables 2 and 3). A possible reason for this discrepancy is that multivariate analysis requires larger sample size. There is currently no accepted molecular typing and biomarker-driven personalized therapy available for HCC patients, and the great heterogenecity demands a systemic therapy designed to meet specific patients' needs rather than serve all [15].

In this study, we proposed a very simple scoring system to evaluate treatment response and survival based on three routine clinical characteristics. This study could be useful for clinicians counseling patients and making an individualized treatment decision for the patient and also may support later guidelines. It indicated that we may use several clinical characteristics to predict the treatment outcome and survival, which might allow for an early change of chemotherapy regimen or treatment strategy in nonresponsive patients. Although our model is simple and efficient, the results were obtained from a small sample group and it's a retrospective study design. It is therefore important to continue researching its specific mechanism and we plan to design a prospective study with expanding the scale of patients. Congruent results also need external validation with independent data sets and determination of its application in advanced hepatocellular carcinoma.

Acknowledgements: This work was supported by a grant from the training program for outstanding young teachers of Jilin University and the project from the Education Department of Jilin Province.

\section{References}

[1] A new prognostic system for hepatocellular carcinoma: a retrospective study of 435 patients: the Cancer of the Liver Italian Program (CLIP) investigators. Hepatology. 1998; 28: 751-5. https://doi.org/10.1002/hep.510280322

[2] ALTEKRUSE SF, MCGLYNN KA, REICHMAN ME. Hepatocellular carcinoma incidence, mortality, and survival trends in the United States from 1975 to 2005. J Clin Oncol 2009; 27: 1485-1491. https://doi.org/10.1200/JCO.2008.20.7753

[3] CHENG AL, KANG YK, CHEN Z, TSAO CJ, QIN S et al. Efficacy and safety of sorafenib in patients in the Asia-Pacific region with advanced hepatocellular carcinoma: a phase III randomised, double-blind, placebo-controlled trial. Lancet Oncol 2009; 10: 25-34. https://doi.org/10.1016/S14702045(08)70285-7
[4] LLOVET JM, RICCI S, MAZZAFERRO V, HILGARD P, GANE E et al. Sorafenib in advanced hepatocellular carcinoma. N Engl J Med 2008; 359: 378-390. https://doi. org/10.1056/NEJMoa0708857

[5] CHEN PJ, FURUSE J, HAN KH, HSU C, LIM HY et al. Issues and controversies of hepatocellular carcinoma-targeted therapy clinical trials in Asia: experts' opinion. Liver Int 2010; 30: 1427-1438. https://doi.org/10.1111/j.14783231.2010.02292.x

[6] CHENG AL, KANG YK, LIN DY, PARK JW, KUDO M et al. Sunitinib versus sorafenib in advanced hepatocellular cancer: results of a randomized phase III trial. J Clin Oncol 2013; 31: 4067-4075. https://doi.org/10.1200/JCO.2012.45.8372

[7] ZHU AX. Systemic therapy of advanced hepatocellular carcinoma: how hopeful should we be? Oncologist 2006; 11: 790-800. https://doi.org/10.1634/theoncologist.11-7-790

[8] QIN S, BAI Y, LIM HY, THONGPRASERT S, CHAO Y et al. Randomized, multicenter, open-label study of oxaliplatin plus fluorouracil/leucovorin versus doxorubicin as palliative chemotherapy in patients with advanced hepatocellular carcinoma from Asia. J Clin Oncol 2013; 31: 3501-3508. https:// doi.org/10.1200/JCO.2012.44.5643

[9] ZAANAN A, WILLIET N, HEBBAR M, DABAKUYO TS, FARTOUX L et al. Gemcitabine plus oxaliplatin in advanced hepatocellular carcinoma: a large multicenter AGEO study. J Hepatol 2013; 58: 81-88. https://doi.org/10.1016/j. jhep.2012.09.006

[10] KELLEY RK. Brivanib and FOLFOX in hepatocellular carcinoma: finding the common themes among negative trials. J Clin Oncol 2013; 31: 3483-3486. https://doi.org/10.1200/ JCO.2013.49.7941

[11] NAGAHAMA H, OKADA S, OKUSAKA T, ISHII H, IKEDA $M$ et al. Predictive factors for tumor response to systemic chemotherapy in patients with hepatocellular carcinoma. Jpn J Clin Oncol 1997; 27: 321-324.

[12] IKEDA M, OKUSAKA T, UENO H, MORIZANE C, KOJIMA Y et al. Predictive factors of outcome and tumor response to systemic chemotherapy in patients with metastatic hepatocellular carcinoma. Jpn J Clin Oncol 2008; 38: 675682. https://doi.org/10.1093/jjco/hyn087

[13] SRIMUNINNIMIT V, SRIURANPONG V, SUWANVECHO S. Efficacy and safety of sorafenib in combination with gemcitabine in patients with advanced hepatocellular carcinoma: a multicenter, open-label, single-arm phase II study. Asia Pac J Clin Oncol 2014; 10: 255-260. https://doi. org/10.1111/ajco.12191

[14] HOLLEBECQUE A, MALKA D, FERTE C DUCREUX M, BOIGE V. Systemic treatment of advanced hepatocellular carcinoma: from disillusions to new horizons. Eur J Cancer 2015; 51: 327-339. https://doi.org/10.1016/j.ejca.2014.12.005

[15] WU Q, QIN SK. Features and treatment options of Chinese hepatocellular carcinoma. Chin Clin Oncol 2013; 2: 38. https://doi.org/10.3978/j.issn.2304-3865.2013.09.07 